\title{
Synergistic enhancement of bone formation and healing by stem cell-expressed VEGF and bone morphogenetic protein-4
}

\author{
Hairong Peng, ${ }^{1,2}$ Vonda Wright, ${ }^{2}$ Arvydas Usas, ${ }^{1,2}$ Brian Gearhart, ${ }^{1}$ Hsain-Chung Shen, $, 2,3$ \\ James Cummins, ${ }^{1}$ and Johnny Huard ${ }^{1,2,4}$
${ }^{1}$ Growth and Development Laboratory, Department of Orthopaedic Surgery, Children's Hospital of Pittsburgh, Pittsburgh, Pennsylvania, USA
${ }^{2}$ Department of Molecular Genetics and Biochemistry, University of Pittsburgh, Pittsburgh, Pennsylvania, USA ${ }^{3}$ Department of Orthopaedic Surgery, University of Pittsburgh Medical Center, Pittsburgh, Pennsylvania, USA \\ ${ }^{4}$ Department of Orthopaedic Surgery, Tri-Service General Hospital, Taipei, Taiwan
}

\begin{abstract}
We investigated the interaction between angiogenic and osteogenic factors in bone formation and bone healing with ex vivo gene therapy using muscle-derived stem cells genetically engineered to express human bone morphogenetic protein-4 (BMP4), VEGF, or VEGF-specific antagonist (soluble Flt1). Our results show that although VEGF alone did not improve bone regeneration, it acted synergistically with BMP4 to increase recruitment of mesenchymal stem cells, to enhance cell survival, and to augment cartilage formation in the early stages of endochondral bone formation. These early effects, coupled with accelerated cartilage resorption, eventually led to a significant enhancement of bone formation and bone healing. The beneficial effect of VEGF on bone healing elicited by BMP4 depends critically on the ratio of VEGF to BMP4, with an improper ratio leading to detrimental effects on bone healing. Finally, we show that soluble Flt1 inhibits bone formation elicited by BMP4. Thus, VEGF plays an important role in bone formation elicited by BMP4, and it can significantly enhance BMP4-elicited bone formation and regeneration through multiple mechanisms. This study has important implications for the formulation of new strategies to improve bone healing through increasing mesenchymal stem cell recruitment and survival, in combination with muscle-derived stem cell-based gene therapy.
\end{abstract}

J. Clin. Invest. 110:751-759 (2002). doi:10.1172/JCI200215153.

\section{Introduction}

Bone regeneration needs to be enhanced for healing large bone defects secondary to tumor or trauma and for treating fracture-delayed unions or nonunions, which constitute $10-15 \%$ of the approximately 6.5 million bone fractures suffered annually in the US (1). Various growth factors have been investigated for their capacity to improve bone healing, and bone morphogenetic proteins (BMPs) are among the most promising. Osteogenic BMPs, including BMP2, BMP4, and BMP7, have been used to induce bone formation and to repair bone defects in various animal models (2-5). In recent years, gene therapy has emerged as an effective approach by which to deliver therapeutic proteins in a more physiological and persistent manner than

Received for publication January 28, 2002, and accepted in revised form July 16, 2002.

Address correspondence to: Johnny Huard, Departments of Orthopaedic Surgery, Molecular Genetics and Biochemistry, and Bio-engineering, University of Pittsburgh, 4151 Rangos Research Center, Children's Hospital of Pittsburgh, 3705 Fifth Avenue, Pittsburgh, Pennsylvania 15213, USA. Phone: (412) 692-7807; Fax: (412) 692-7095; E-mail: jhuard+@ pitt.edu.

Conflict of interest: Johnny Huard is a consultant in a sponsored research agreement with Cook MyoSite.

Nonstandard abbreviations used: bone morphogenetic protein (BMP); muscle-derived stem cell (MDSC); soluble Flt1 (sFlt1); post-implantation (PI). was previously anticipated (6-14). Although osteogenic BMP alone is sufficient to induce bone formation, this process appears to involve several different pathways acting in concert. One of these is angiogenesis, since a compound of antiangiogenic activity hampers ectopic bone formation induced by BMP2 (15).

VEGF, the best-characterized angiogenic factor, plays an important role in bone growth via the endochondral ossification pathway. Blocking VEGF leads to a decrease in trabecular bone formation at the growth plate secondary to suppression of blood vessel invasion and impairment of cartilage resorption (16). The involvement of VEGF in bone formation also is suggested by its interaction with humoral factors that regulate bone homeostasis (17). Despite the close association between VEGF and bone development indicated by the loss-of-function approach discussed above (16), it remains unknown whether VEGF function is essential for bone formation induced by osteogenic BMPs. We also do not know whether supplying extra VEGF (i.e., the gain-of-function approach) can further enhance the bone formation or bone healing process, with or without the presence of BMPs. Resolving this issue is essential for development of better strategies to improve bone healing. Furthermore, it is unclear whether VEGF, in addition to its integral role in cartilage resorption during 
endochondral bone formation (16), also influences other steps in bone formation and bone healing processes. To address these important questions, we used an ex vivo gene therapy approach based on muscle-derived stem cells (MDSCs) genetically engineered to express human BMP4, human $\mathrm{VEGF}_{165}$, or the VEGF antagonist soluble Flt1 (sFlt1). We then studied the effect of VEGF and VEGF antagonist on BMP4-induced bone formation and bone healing and attempted to outline the related cellular and molecular processes. Finally, we investigated whether it is possible to enhance the beneficial action and prevent the previously reported adverse effects associated with hemangioma formation (18-20) by controlling the ratio of VEGF to BMP4. Our studies led to a series of discoveries that provide new insight into the interaction between angiogenic factor VEGF and osteogenic factor BMP4 during the processes of bone formation and bone regeneration and thus have important implications for the formulation of new therapeutic strategies to enhance bone repair.

\section{Methods}

Construction of retroviral vectors expressing human BMP4 and VEGF. Human BMP4 and BMP2 cDNAs were amplified by PCR from the respective phage clones (American Type Culture Collection, Manassas, Virginia, USA). A Kozak sequence was introduced to the $5^{\prime}$ end of each cDNA to enhance protein expression in mammalian cells. BMP4-1 (CCGCTCGAGGCGGCCGCCCACCATGCTGATGGTCGTTTTATTATG) and BMP4-2 (TCCATCGATAGATCTATCCTCAAGGACTGCCTG) primers were used to generate the BMP4 expression construct. Similarly, BMP2-1 (CCGCTCGAGGCGGCCGCCCACCATGGTGGCCGGGACCCGCTGTCT) and BMP2-2 (TCCATCGATAGATCTGCTGTACTAGCGACACCCACA) primers were used to amplify the BMP2 construct. Following cloning into pBluescript II KS(-), sequences of selected clones were verified by sequencing both strands of $\mathrm{cDNA}$.

The BMP2/4 hybrid construct, in which the original sequence coding for the propeptide of BMP4 was replaced by the corresponding propeptide sequence of the BMP2 CDNA, was generated as described previously (11). This modification led to a markedly higher level of BMP4 secretion in transfected cells (11). The modified BMP4 cDNA was subcloned into retroviral vector pCLX, derived from pLXSN (from A. Dusty Miller, Fred Hutchinson Cancer Research Center, Seattle, Washington, USA) by removing the SV40 promoter and the neomycin-resistant gene, and replacing the $\mathrm{U} 3$ in the $5^{\prime}$ long-terminal repeat with the human cytomegalovirus promoter. The resultant vector was termed PCLBMP4. Retroviral vectors expressing human VEGF, sFlt1, or the bacterial LacZ gene were constructed by cloning the VEGF $_{165}$ cDNA (from InvivoGen, San Diego, California, USA), cDNA encoding human sFlt1 (from InvivoGen), or the LacZ gene into pCLX, resulting in pCLVEGF, pCLsFlt1, and pCLLacZ, respectively.
Vector DNA was converted into replication-defective retrovirus by cotransfection, with calcium-phosphate precipitation, into packaging cell line GP-293 (CLONTECH Laboratories Inc., Palo Alto, California, USA). pVSVG, a plasma that expressed vascular stomatitis virus glycoprotein, was used to provide the viral envelope. The titer of the viral vectors was estimated to be $5 \times 10^{5}$ to $2 \times 10^{6} \mathrm{CFUs} / \mathrm{ml}$ by limiting dilution. The retroviruses expressing BMP4, VEGF, sFlt1, or LacZ were termed retroBMP4, retroVEGF, retrosFlt1, and retroLacZ, respectively.

Cell culture and transduction. The MDSCs (MC13 clone) were isolated and cultured as previously described (10). These cells were transduced separately with retroviral vectors at an moi of 5 in the presence of $8 \mu \mathrm{g} / \mathrm{ml}$ polybrene. The transduced cells were expanded for 2 weeks before being used in animal experiments. At the end of expansion, cells and the conditioned media were sampled to determine the level of transgene expression.

BMP4 bioassay, Western blot, and ELISA. The level of functional BMP4 secreted from the transduced cells was estimated with a BMP4 bioassay and Western blot as described previously (11). The levels of VEGF or sFlt secreted from the transduced cells were measured using ELISA kits (R\&D Systems Inc., Minneapolis, Minnesota, USA).

Ectopic bone formation. All the animal experiments were approved by the Animal Research and Care Committee of the Children's Hospital of Pittsburgh (Protocol no. 02/01). C57BL/6J mice (male, 12 weeks old) were randomly divided into different groups as detailed in Results. Under anesthesia, an $8-\mathrm{mm}$ incision was made over the lateral aspect of each femur, a 7-mm Gelfoam disk (Pharmacia \& Upjohn Co., Kalamazoo, Michigan, USA) impregnated with a designated number of transduced cells was implanted into an intramuscular pocket created by blunt dissection, and the wound was closed with suture. Ectopic bone formation was monitored radiographically and histologically at $3,7,10,14$, and 28 days post-implantation (PI). The samples taken at 14 and 28 days were divided into two equal parts: one part for histologic analysis, another part for determination of $\mathrm{Ca}^{++}$content and alkaline phosphatase activity. The relative bone area and bone density detected by radiography were quantified with Northern Eclipse version 6.0 software (Empix Imaging Inc., North Tonawanda, New York, USA). Statistical differences between groups were analyzed using a Student's $t$ test with Microsoft Excel (Microsoft Corp., Seattle, Washington, USA).

Skull defect healing. C57BL/6J mice (male, 12 weeks old) were randomly divided into designated groups. Under anesthesia, a 6-mm-diameter defect was created in the parietal bone without breaching the dura, a 7-mm Gelfoam disk impregnated with a designated number of transduced cells was implanted into the defect, and the wound was closed with suture. Bone healing was monitored radiographically and histologically at designated time points after surgery. 

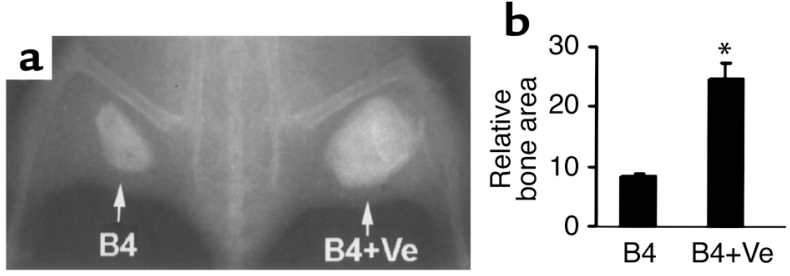

BMP4
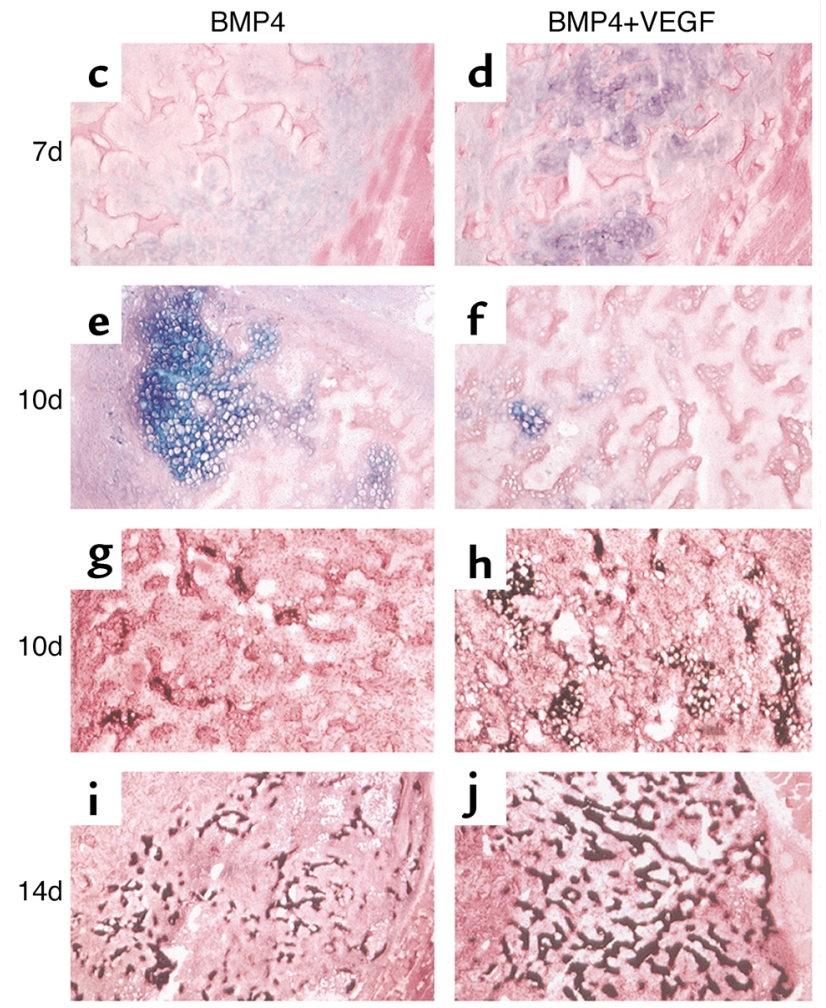

Quantitative analyses of bone area and bone density were performed as described above.

Histologic analysis. von Kossa staining, which stains the phosphates and carbonates deposited in mineralized bone, was performed as described previously (10). For Alcian Blue staining, the samples were fixed in $4 \%$ formaldehyde for 10 minutes, stained in the solution for 30 minutes, counterstained in eosin, and mounted. The slides were visualized by light microscopy (E-800; Nikon Inc., Melville, New York, USA). The area of cartilage formation was measured using Northern Eclipse version 6.0 software after image digitization.

\section{Figure 2}

VEGF enhances angiogenesis during bone formation elicited by MDSCs expressing BMP4. (a and b) CD31 immune staining shows more active capillaries (arrows) branching from the surrounding muscle and growing toward the scaffolds (S) in the BMP4+VEGF group than in the BMP4 group at 4 days PI. (c and $\mathbf{d}$ ) An extensive network of capillaries (arrows) formed inside the scaffolds of the BMP4+VEGF group, while only a modest level of capillaries formed in the scaffolds of the BMP4 group, at 10 days. (e) Histomorphometry demonstrates that the relative capillary density was significantly higher in the BMP4+VEGF group than in the BMP4 group $\left({ }^{*} P<0.01\right)$ at 10 days. Magnification: $\mathbf{a}$ and $\mathbf{b}, \times 400 ; \mathbf{c}$ and $\mathbf{d}, \times 100$.

\section{Figure 1}

VEGF enhances endochondral bone formation elicited by MDSCs expressing BMP4. (a) Radiograph shows augmented bone formation in the BMP4+VEGF (B4+Ve) site compared with the BMP4 (B4) site 4 weeks PI. (b) Quantitative analysis shows that the BMP4+VEGF group produced significantly more bone than the BMP4 group $\left({ }^{*} P<0.01\right.$, $n=4)$. (c-f) Alcian Blue staining shows enhanced cartilage formation (purple) at 7 days and accelerated cartilage resorption at 10 days in the BMP4+VEGF group compared with the BMP4 group. ( $\mathbf{g}-\mathbf{j})$ von Kossa staining shows more extensive cartilage mineralization (black) at 10 days and increased mineralized bone formation (black) at 14 days in the BMP4+VEGF group compared with the BMP4 group. Magnification: $\mathbf{c}, \mathbf{d}$, and $\mathbf{g}-\mathbf{j}, \times 100$; $\mathbf{e}$ and $\mathbf{f}, \times 200$.

Immunocytochemical staining for blood vessels. Cryosections of bone samples were stained with a rat antimouse CD31 mAb (clone MEC13.3; PharMingen, San Diego, California, USA) diluted 1:100 with reagents in the VECTASTAIN Elite ABC kit (Vector Laboratories Inc., Burlingame, California, USA) following the protocol recommended by the manufacturer. The sections were counterstained with hematoxylin and eosin. The relative capillary density was estimated by counting the capillary number and measuring the length of the capillaries in the defined area after image digitization.

Apoptosis assay. Bone samples were fixed with $4 \%$ paraformaldehyde, and the apoptotic cells were detected using the In Situ Cell Death Detection kit, POD (Roche Molecular Biochemicals, Mannheim, Germany) according to the instructions provided by the manufacturer. More than 500 cells in each quadruplicated sample were counted to determine the percentage of apoptotic cells in each group. The statistical differences were analyzed using a two-tailed Student's $t$ test.
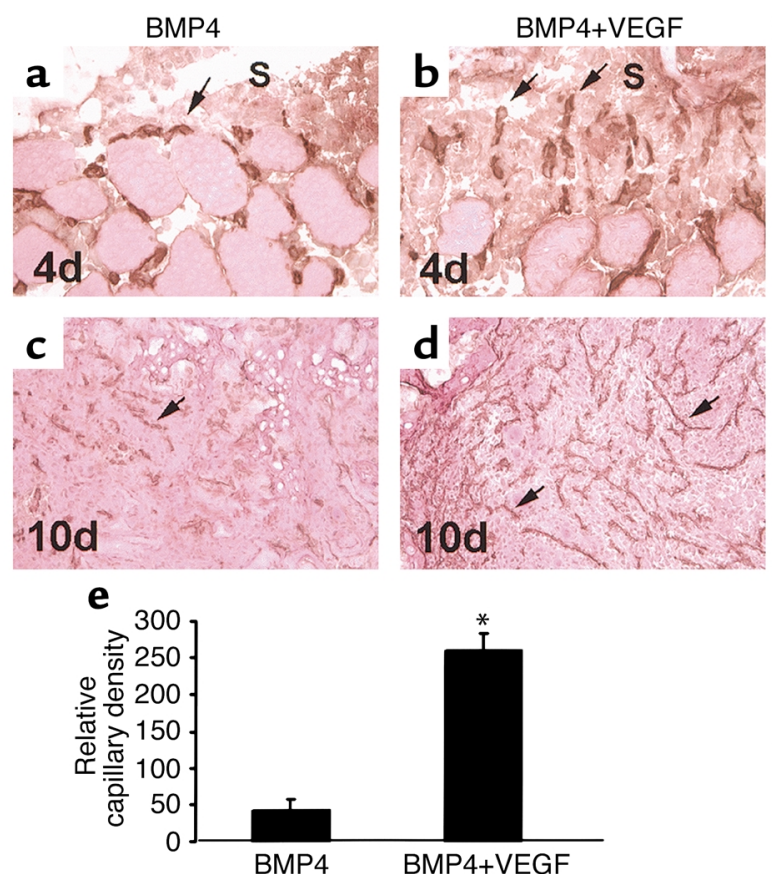

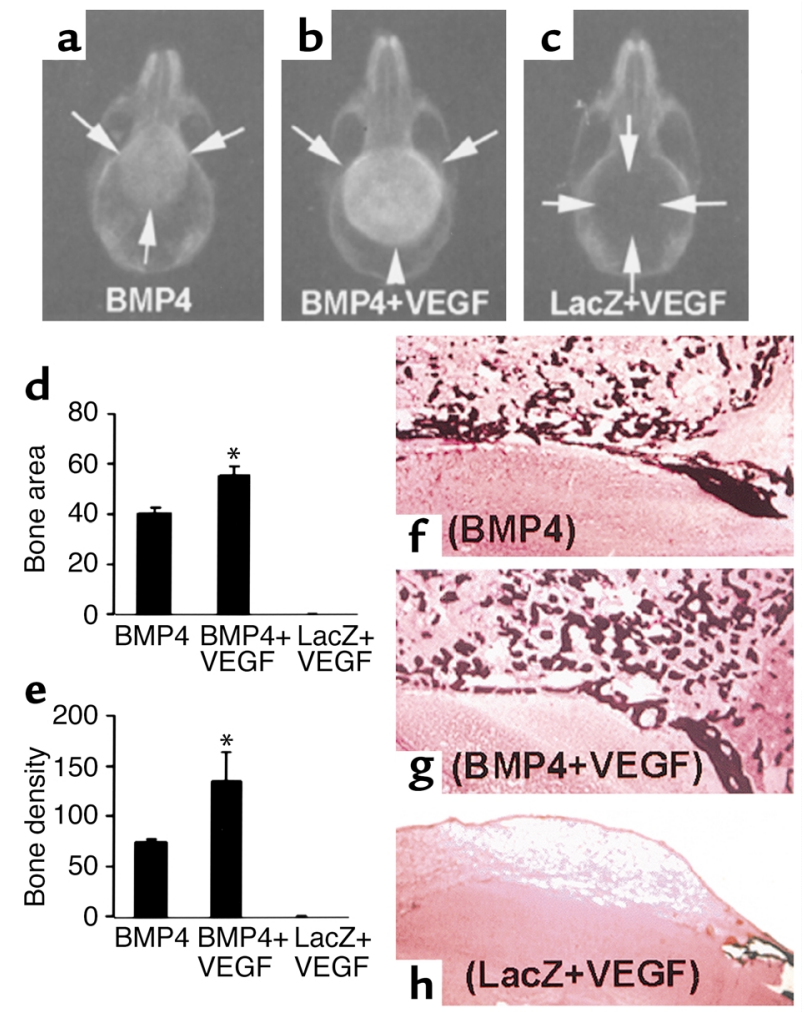

\begin{abstract}
Results
Expression of BMP4 or VEGF from transduced MDSCs. MDSCs were able to synthesize, process, and secrete active human BMP4 following transduction with retroBMP4. The level of BMP4 reached $115 \pm 20$ $\mathrm{ng} / 10^{6}$ cells $/ 24$ hours at 1 week after transduction and persisted for at least 4 weeks in vitro. The secreted BMP4 was biologically active as evidenced by its ability to stimulate alkaline phosphatase activity in $\mathrm{C} 2 \mathrm{C} 12$ cells (data not shown). MDSCs transduced with retroVEGF secreted VEGF at an average level of $214 \pm 35$ $\mathrm{ng} / 10^{6}$ cells $/ 24$ hours over a 4 -week period, whereas cells transduced with retroLacZ did not secrete a detectable level of VEGF (data not shown). RetrosFlttransduced MDSCs secreted sFlt at a level of $20 \pm 5$ $\mathrm{ng} / 10^{6}$ cells $/ 24$ hours.

$V E G F$ enhanced bone formation elicited by MDSCs expressing BMP4. To determine the effects that supplying extra VEGF would have on BMP4-elicited bone formation, we compared bone formation elicited by BMP4expressing cells (BMP4) with that elicited by a mixture
\end{abstract}

\section{Figure 4}

Dose response in bone healing induced by BMP4+VEGF-expressing MDSCs. (a-c) Radiography shows complete bone regeneration induced by two different doses of BMP4+VEGF-expressing cells $(B 4+V e)$. No bone formation occurred in the defects implanted with LacZ+VEGF-expressing cells ( $\mathrm{Lac}+\mathrm{Ve}$ ). The ratio of BMP4- to VEGFexpressing cells was kept at $5: 1$. Arrows mark the edge of the defects or the regenerated bone. ( $\mathbf{d}$ and $\mathbf{e}$ ) Quantitative analysis shows the correlation between the amount of bone regenerated and the quantity of transduced cells implanted $\left({ }^{*} P<0.05, n=4\right)$.

\section{Figure 3}

VEGF enhances healing of critical-sized calvarial defects elicited by MDSCs expressing BMP4. (a-c) Radiography shows complete bone healing of the defects in both the BMP4 and the BMP4+VEGF groups and no bone healing in the LacZ+VEGF group at 3 weeks PI. Arrows mark the edge of defects or newly formed bone. (d and $\mathbf{e}$ ) Quantitative analysis shows a significant enhancement of bone regeneration in the BMP4+VEGF groups relative to the BMP4 group $\left({ }^{*} P<0.05, n=4\right)$. $(\mathbf{f}-\mathbf{h})$ von Kossa staining reveals coarser mineralized bone trabeculae in the BMP4+VEGF group than in the BMP4 group. No mineralized bone formed in the defect of the LacZ+VEGF group. Magnification: $\mathbf{f}-\mathbf{h}, \times 40$.

of BMP4- and VEGF-expressing cells (BMP4+VEGF), with the same number of cells being implanted in the same animals to rule out variations. We chose a ratio of 5:1 (BMP4-/VEGF-expressing cells) based on the rationale that a high dose of VEGF might lead to detrimental effects due to the formation of hemangioma, as previously described (18-20). Radiography showed that the BMP4+VEGF produced significantly more bone than the BMP4 did at 4 weeks PI (Figure 1, a and b). These results were confirmed by biochemical analysis of calcium content and alkaline phosphatase activity of the newly formed bone (not shown).

VEGF enhanced cartilage formation and accelerated cartilage resorption in endochondral bone formation induced by BMP4. Histologic analysis of the stages of bone formation showed increased cartilage formation in the BMP4+VEGF group compared with the BMP4 group at 7 days PI (Figure $1, c$ and d), with a significant difference in the relative cartilage areas $(399 \pm 65$ vs. $186 \pm 29$, $P=0.01, n=4)$. Interestingly, cartilage resorption was accelerated in the BMP4+VEGF group, leading to more extensive mineralization than in the BMP4 group (Figure $1, \mathrm{e}-\mathrm{h}$ ) at 10 days PI. At 14 days PI, trabecular bone formation was increased in the BMP4+VEGF group,

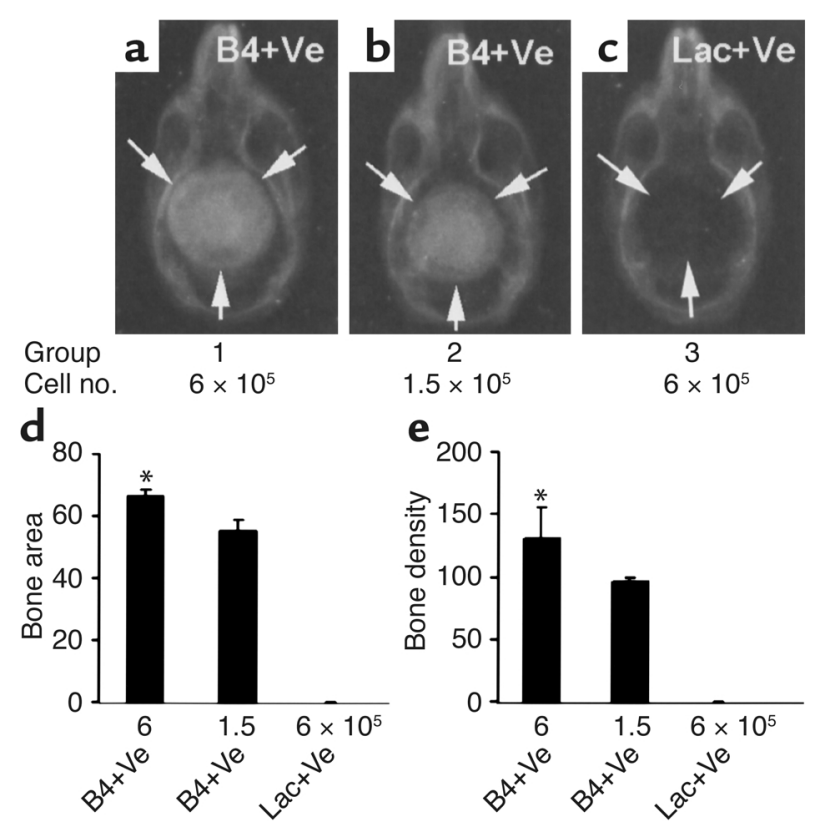



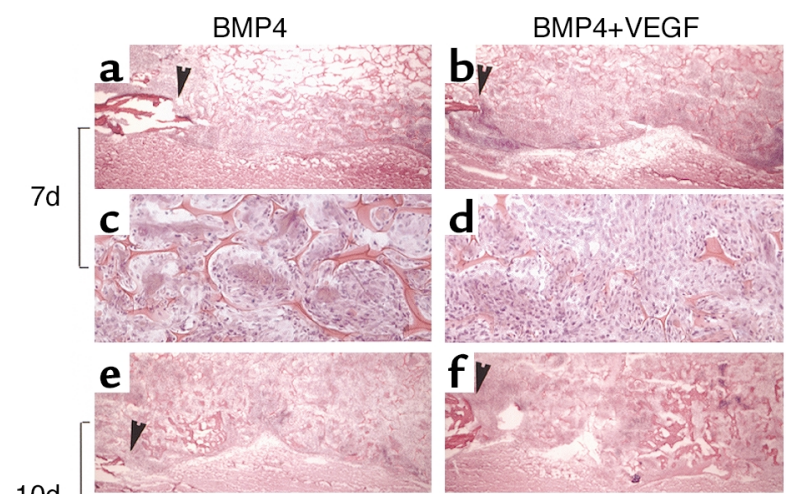

$10 \mathrm{~d}$
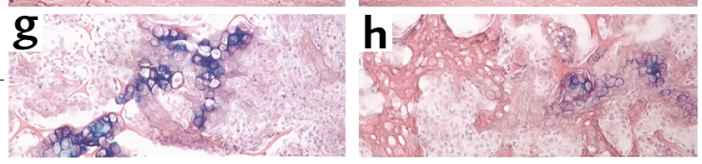

$10 d$
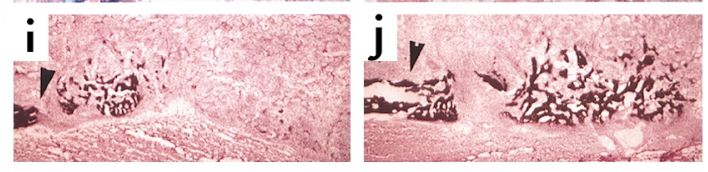

$14 d$
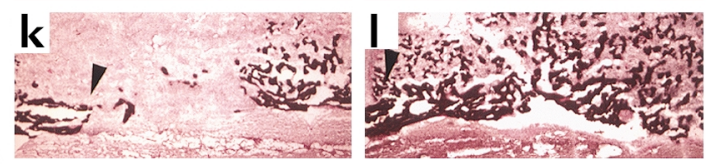

compared with the BMP4 group (Figure 1, i and $j$ ). Notably, the bone formed in the BMP4+VEGF group consisted of denser and better-organized trabeculae, indicating bone of higher quality.

$V E G F$ enhanced angiogenesis in bone formation induced by MDSCs expressing BMP4. Angiogenesis during bone formation induced by MDSCs expressing BMP4 with or without VEGF was investigated using immunohistochemistry for CD31, a specific tissue marker of endothelial cells (21). Angiogenesis was more active in the tissues of the BMP4+VEGF group than in those of the BMP4 group; there were significantly more capillaries branching from the surrounding muscle and growing toward the scaffolds at 4 days PI (Figure 2, a and b), as well as increased mesenchymal cell infiltration and enhanced capillary ingrowth at 7 days PI (not shown). By 10 days PI, a much more extensive capillary network was formed in the BMP+VEGF group than in the BMP4 group (Figure 2, c and d), with a fivefold increase in the relative capillary density (Figure $2 \mathrm{e} ; P<0.01)$. Angiogenesis in both groups subsided at 14 days PI (not shown).

VEGF enhanced healing of skull defects induced by MDSCs expressing BMP4. To determine the effects of VEGF on BMP4-induced bone healing, critical-sized calvarial defects were treated with collagen disks impregnated with BMP4-expressing cells alone $\left(6 \times 10^{5}\right)$, both BMP4expressing and VEGF-expressing cells $\left(5 \times 10^{5}: 1 \times 10^{5}\right)$, or LacZ-expressing and VEGF-expressing cells $\left(5 \times 10^{5}\right.$ : $\left.1 \times 10^{5}\right)$. The defects were completely healed at 3 weeks $\mathrm{PI}$ in all eight animals in the BMP4 and BMP4+VEGF groups; in contrast, no bone healing occurred in the LacZ+VEGF group (Figure 3, a-c). The bone formed in

\section{Figure 5}

Critical-sized calvarial defects healed through the endochondral ossification pathway in both BMP4 and BMP4+VEGF groups. (a-h) Alcian Blue staining shows cartilage formation in the defects implanted with transduced MDSCs. The edges of the bone defects are marked by arrowheads. Mesenchymal cell infiltration was more abundant in the BMP4+VEGF group (b and $\mathbf{d}$ ) than in the BMP4 group ( $\mathbf{a}$ and $\mathbf{c}$ ) at 7 days Pl. $\mathbf{c}$ and $\mathbf{d}$ display local magnifications of $\mathbf{a}$ and $\mathbf{b}$, respectively. At 10 days, cartilage bridging the defects was mostly resorbed, leaving traces of hypertrophic chondrocytes $(\mathbf{e}-\mathbf{h})$. $\mathbf{g}$ and $\mathbf{h}$ display local magnifications of $\mathbf{e}$ and $\mathbf{f}$, respectively. (i-I) von Kossa staining demonstrates coupled bone mineralization at 10 days and increased mineralized bone formation at 14 days in the BMP4+VEGF group ( $\mathbf{j}$ and $\mathbf{I}$ ) compared with the BMP4 group (i and k). Magnification: $\mathbf{a}, \mathbf{b}, \mathbf{e}, \mathbf{f}$, and $\mathbf{i}-\mathbf{I}, \times 40 ; \mathbf{c}, \mathbf{d}, \mathbf{g}$, and $\mathbf{h}, \times 200$.

the BMP4+VEGF group was significantly larger and denser than the bone formed in the BMP4 group (Figure $3, \mathrm{~d}$ and e) at 3 weeks PI. A similar trend was observed at 6 weeks PI (not shown). These radiographic results were confirmed by histologic analysis, which showed complete bone union of the defects in the BMP4 and BMP4+VEGF groups, with no bone healing in the LacZ+VEGF group (Figure 3, $\mathrm{f}-\mathrm{h}$ ).

We next explored whether it is possible to heal the critical-sized calvarial defects with a reduced number of transduced MDSCs. As shown in Figure 4, complete bone healing was elicited even with a fourfold reduction in the number of transduced cells. Furthermore, the amount of bone formed correlated positively with the number of cells implanted (Figure 4, d and e). It also was noted that the degree of bone healing elicited with $1.5 \times 10^{5}$ BMP4+VEGF-expressing cells was comparable to that elicited by a fourfold greater number of cells expressing BMP4 alone (compare Figure 4b with Figure 3a).

Healing of critical-sized calvarial defects elicited by BMP4 or $B M P 4+V E G F$ was mediated through endochondral ossification pathway. Calvarial bone is developed through the intramembranous pathway; non-critical-sized calvarial defects are normally healed through the same pathway. Surprisingly, however, the healing of critical-sized calvarial defects elicited by both BMP4- and BMP4+VEGFexpressing MDSCs was mediated primarily through the endochondral bone formation pathway (Figure 5, a-l), a process mimicking that of ectopic bone formation in skeletal muscle described previously (Figure 1).

\section{Table 1}

VEGF enhances cell recruitment and cell survival in bone regeneration elicited by MDSCs expressing BMP4

\begin{tabular}{lccc} 
& BMP4 & VEGF+BMP4 & VEGF \\
Total cells & $396 \pm 24$ & $628 \pm 43$ & $169 \pm 19$ \\
Apoptotic cells & $277 \pm 35$ & $24 \pm 3$ & ND \\
\hline
\end{tabular}

Cell numbers were obtained by counting the cells in a photographic field with magnification of $\times 20$ objective. Five representative fields were counted in each section, and five sections of each sample were examined. Total cells: BMP4 vs. VEGF+BMP4, $P=0.01$; BMP4 vs. VEGF, $P<0.01$. Apoptotic cells: BMP4 vs. VEGF+BMP4, $P<0.01$. ND, not determined. 

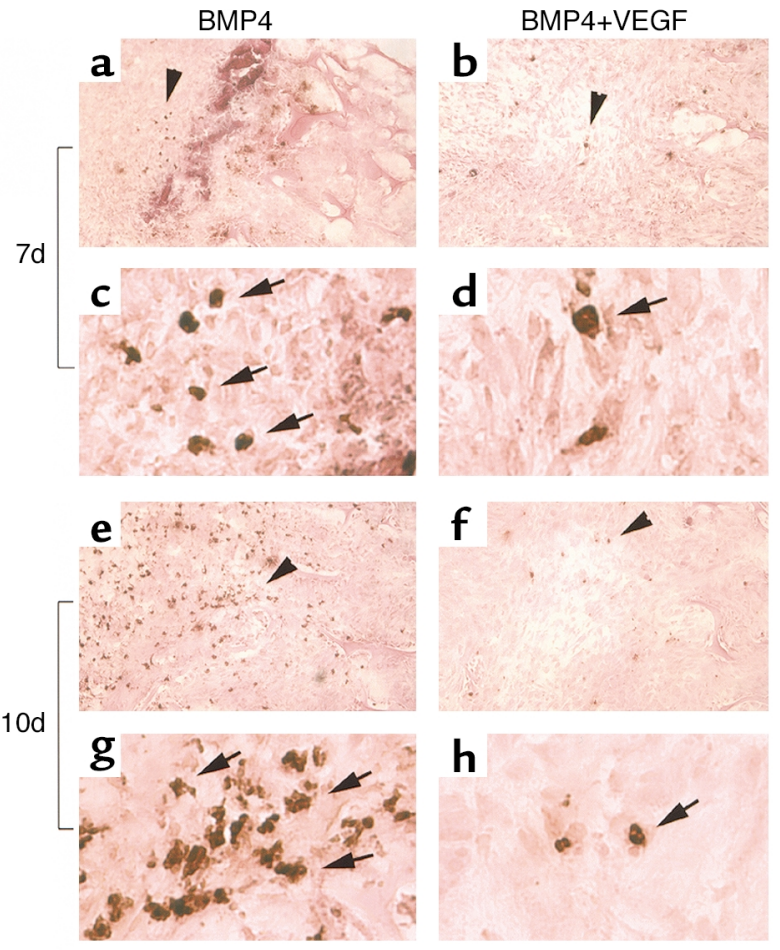

VEGF increased cell recruitment and cell survival in bone regeneration elicited by MDSCs expressing BMP4. To explore mechanisms that may account for the increased cartilage formation induced by VEGF during endochondral bone formation elicited by MDSCs expressing BMP4, we assessed the relative number of cells recruited to the bone healing site 7 days PI, when the distribution of the cells in the bone regeneration site is relatively even and cells can be counted accurately. A significant increase in mesenchymal cell recruitment was noted in the BMP4+VEGF group, as compared with the BMP4 group (Table 1). We then determined whether VEGF can enhance cell survival at the bone regeneration sites. Apoptosis assay showed that the number of apoptotic

\section{Figure 7}

The proper ratio of VEGF to BMP4 is critical to ensure a beneficial effect on bone healing. In groups 1-3, the ratios of VEGF- to BMP4expressing cells were $0.2,1$, and 5 , respectively; in group 4 , only VEGF-expressing cells were used. Arrows mark the edge of the bone defects and regenerated bone. (a-d) Radiography shows complete bone healing in groups 1 and 2, partial bone healing in group 3, and no bone healing in group 4. (e-h) von Kossa staining shows regeneration of cortical bone-like structure (arrow) in group 1 (e) but not in other groups, although bone union also occurred in group $2(\mathbf{f})$. Bone regeneration was significantly reduced in group 3 as compared with groups 1 and $2(\mathbf{e}-\mathbf{g})$. Only fibrous tissue fills the defect in group $4(\mathbf{h})$. Arrowheads mark the edge of the critical-sized bone defects and the regenerated bone. Magnification: $\times 40$. ( $\mathbf{i}$ and $\mathbf{j}$ ) Quantitative analysis shows a significant decrease in bone regeneration in group 3 as compared with groups 1 and 2, while no bone formation was detected in the group containing only VEGF-expressing cells. Group 1 vs. group $2, P>0.05, n=4$; group 1 vs. group 3 , $P<0.01, n=4 ;{ }^{*} P<0.01$ when compared to group 1 .

\section{Figure 6}

VEGF increases cell survival in the bone regeneration site of criticalsized calvarial defects. TUNEL assay shows a reduction in apoptotic cells (with brown-stained nuclei as indicated by arrows) in the BMP4+VEGF group compared with the BMP4 group at 7 days (a-d) and, more strikingly, at 10 days $\mathrm{PI}(\mathbf{e}-\mathbf{h}) . \mathbf{c}, \mathbf{d}, \mathbf{g}$, and $\mathbf{h}$ display higher magnifications of $\mathbf{a}, \mathbf{b}, \mathbf{e}$, and $\mathbf{f}$, respectively, at the area indicated by arrowheads. Magnification: a, b, e, and f, $\times 200 ; \mathbf{c}, \mathbf{d}, \mathbf{g}$, and $\mathbf{h}, \times 1,200$.

cells was reduced slightly at 7 days and significantly at 10 days PI in the BMP4+VEGF group compared with the BMP4 group (Figure 6, a-h; Table 1).

Proper ratio of VEGF to BMP4 is critical to ensure synergistic effects in bone healing. To determine the optimal ratio of VEGF to BMP4 for improving the healing of criticalsized defects, such defects were implanted with a fixed number of BMP4-expressing cells $\left(1 \times 10^{5}\right)$ supplemented with variable numbers of VEGF-expressing cells $\left(2 \times 10^{4}, 1 \times 10^{5}\right.$, and $5 \times 10^{5}$ in groups 1,2 , and 3 , respectively). The ratios of VEGF to BMP4 in these three groups were thus $0.2,1$, and 5 , respectively. The total cell number in each group was brought to $6 \times 10^{5}$ by adding LacZ-expressing cells to rule out variation secondary to cell number differences. Group 4 was implanted with VEGF-expressing cells only $\left(5 \times 10^{5}\right)$. Three weeks PI, the bone defects were healed in all mice of groups 1 and 2 , partially healed in group 3 mice, and unhealed in group 4 mice (Figure 7, a-d, i, and j). These radiographic results were confirmed by histologic analysis (Figure $7, \mathrm{e}-\mathrm{h}$ ), which showed complete bone union in groups 1 and 2, with the quality of healing being superior in group 1 due to better bony bridging of the defect. In contrast, only

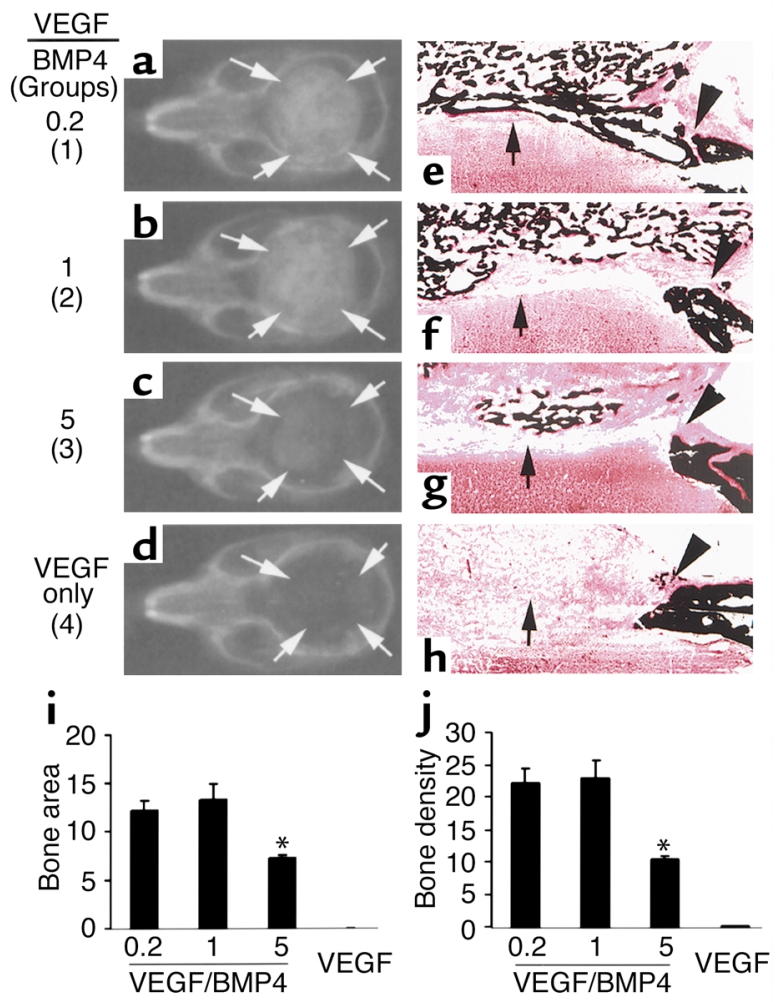



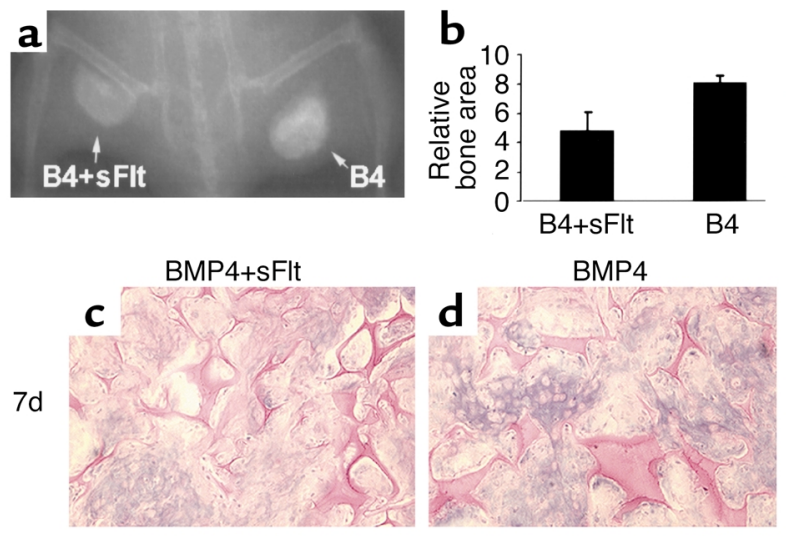

$10 \mathrm{~d}$
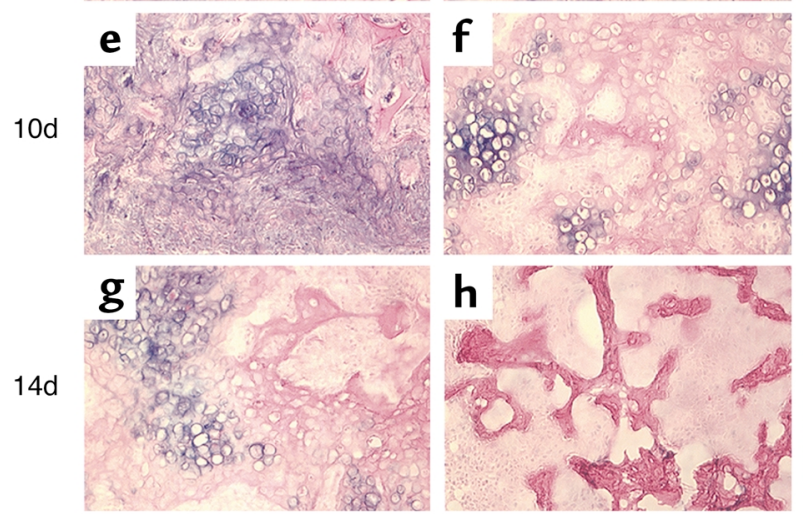

Figure 8

VEGF antagonist inhibits bone formation elicited by MDSCs expressing BMP4. (a) Radiography shows decreased bone formation in the BMP4+sFlt1 (B4+sFlt) site compared with the BMP4 (B4) site 4 weeks $\mathrm{PI}$. (b) Quantitative analysis shows a significant difference in relative bone area between the BMP4+sFlt and BMP4 groups $\left({ }^{*} P<0.05\right)$. (c-h) Alcian Blue staining shows reduced cartilage formation at 7 days and delayed cartilage resorption at 10 and 14 days in the BMP4+sFlt group compared with the BMP4 group. Magnification: $\times 200$.

small patches of mineralized bone formed in group 3. The rest of the tissue filling the defects consisted primarily of soft tissue rich in capillaries (not shown). There was no mineralized bone formation in the control group implanted with only a high dose of VEGF-expressing cells. Thus, neither high nor low doses of VEGF alone improved healing of critical-sized bone defects.

VEGF antagonist inbibited bone formation elicited by MDSCs expressing BMP4. To determine whether intrinsic VEGF activity is important for bone formation elicited by MDSCs expressing BMP4, we examined the effect of a VEGF-specific antagonist (sFlt1). Bone formation was significantly inhibited in all five animals at the sites implanted with $1 \times 10^{5}$ MDSCs expressing BMP4 and $2 \times 10^{5}$ MDSCs expressing sFlt 1 (BMP4+sFlt), compared with the sites implanted with the same number of MDSCs expressing BMP4 (BMP4) plus $2 \times 10^{5}$ MDSCs expressing LacZ (Figure 8 , a and b). Histologic analysis revealed that sFlt1 reduced cartilage formation and delayed cartilage resorption during endochondral bone formation elicited by MDSCs expressing BMP4 (Figure 8, c-h).

\section{Discussion}

Our results demonstrate that VEGF activity is required for optimal bone formation elicited by MDSCs expressing BMP4. More importantly, our findings show that VEGF and BMP4 act synergistically to enhance both bone formation and bone healing. In addition to its effects on enhancing angiogenesis and accelerating cartilage resorption, VEGF in the presence of BMP4 promotes recruitment of mesenchymal cells to bone formation and regeneration sites and enhances cell survival, subsequently leading to augmented cartilage formation. Thus, VEGF enhances BMP4-elicited endochondral bone formation not only by influencing steps after cartilage formation, but also by affecting steps in the early stages of the bone formation cascade. Intriguingly, our study demonstrates that the beneficial effect of VEGF on bone healing depends critically on its ratio to BMP4, with improper proportions leading to detrimental effects on the healing process. Finally, this study shows that the critical-sized calvarial defects were healed via the unexpected endochondral instead of the default intramembranous ossification pathway, following treatment with MDSCs expressing BMP4. Such pathway switching may be attributed to the use of MDSCs or BMP4.

VEGF is important for both early and later stages of endochondral bone formation. The role of VEGF in bone formation elicited by BMPs is still unknown. Our study demonstrates that VEGF function appears to be critical not only for cartilage resorption, but also for the initiation of endochondral bone formation. This finding is supported by the ability of VEGF-specific antagonist (sFlt1) to inhibit bone formation by reducing cartilage formation and delaying cartilage resorption in the endochondral ossification pathway. Thus, the role of VEGF in the later stages of bone formation elicited by BMP4 is similar to its role in postnatal bone growth (16). The effect of VEGF on the early stages of bone formation, however, has not, to our knowledge, been described previously.

VEGF acts synergistically with BMP4 to improve bone formation and bone healing through multiple mechanisms. The importance of VEGF in the early stages of bone formation is further supported by our results demonstrating synergy between BMP4 and VEGF in improving bone formation and bone healing. In addition to accelerated cartilage resorption due to enhanced angiogenesis, our study reveals much more extensive effects of VEGF on the early stages of bone formation. These include increased recruitment of mesenchymal stem cells to the bone formation and regeneration site, enhanced cell survival, and increased cartilage formation. We believe these early effects are those primarily responsible for the overall significant enhancement in bone formation, because accelerated cartilage resorption alone is unlikely to account for such an outcome.

VEGF may act indirectly or directly to increase recruitment of mesenchymal stem cells. The indirect effects stem from its ability to increase vascular permeability 
$(22,23)$, which may facilitate migration of host mesenchymal stem cells to the bone regeneration site. VEGF may also directly attract mesenchymal stem cells to the bone regeneration site. This possibility is supported by the expression of the VEGF receptor Flk 1 on some stem cells, including MDSCs (10). Furthermore, a recent study indicates that VEGF may serve as a chemoattractant involved in the developmental migration of Drosophila blood cells (24). Currently, we are investigating whether VEGF can serve as a chemoattractant for mammalian hematopoietic stem cells or mesenchymal stem cells.

It is intriguing that VEGF is capable of enhancing cell survival at the bone regeneration sites. Such an effect may be attributed to enhanced circulation due to active angiogenesis mediated by VEGF. Alternatively, VEGF also may exert direct antiapoptotic effects on the bone-forming cells in a manner similar to that in which it affects endothelial cells or leukemia cells (25). Since apoptosis is associated with differentiation of osteoblasts induced by BMP (26) and occurs naturally during normal fracture healing (27), our results suggest that supplying VEGF to counteract these proapoptotic effects could augment bone formation and bone healing.

The synergistic effect of VEGF on BMP4-elicited bone formation also highlights the insufficiency of the intrinsic angiogenic effect of BMP4 for optimal bone induction - although a similar protein, BMP2, has been found to display angiogenic activity (14). Similarly, the ability of exogenous VEGF to enhance bone regeneration elicited by BMP4 also indicates that the amount of endogenous VEGF is not sufficient for optimal bone healing, even though VEGF can be produced by a variety of cells involved in bone formation $(17,28,29)$.

VEGF alone is not sufficient to improve bone healing of critical-sized defects. It was surprising that MDSCs expressing VEGF alone did not improve bone healing. Such a failure to enhance bone healing was not due to improper dosage, as both low and high doses of VEGF-expressing cells were examined. Thus it appears that, although VEGF can affect osteogenic cells $(17,29,30)$ and is essential for bone growth (16), supplying VEGF alone is not sufficient to initiate the cascade of bone regeneration. Interestingly, we also observed that high doses of MDSCs expressing VEGF alone did not cause further damage to the bone defect. This finding contradicts previous reports indicating that high doses of VEGF lead to hemangioma $(18,19)$ or disruption in embryonic myocardium development (20). Such conflicting results may be attributable to the use of different animals or animals at different development stages (e.g., immunedeficient animals [ref. 18] or animals in early stages of development [ref. 20]).

Proper ratio of VEGF to BMP4 is critical to ensure synergistic effects and prevent detrimental reaction in bone healing. In accordance with our findings regarding the interaction between VEGF and BMP4 in the promotion of bone healing, we also discovered that the ratio of VEGF to BMP4 is critical to ensure the enhancement of bone healing elicited by transduced MDSCs. A synergistic effect in bone regeneration occurred when the number of VEGF-expressing cells was one-fifth that of the BMP4-expressing cells. In contrast, reversing that ratio to 5:1 significantly impaired bone regeneration. The amount of bone formed in the latter group was significantly reduced compared with that elicited by the same number of BMP4-expressing cells alone (data not shown). Thus, excessive VEGF in relation to BMP4 leads to an impairment in bone formation, possibly by pushing mesenchymal stem cells toward an endothelial lineage, thereby reducing the number of cells available for chondrogenic and osteogenic differentiation. Indeed, in the tissue filling in the bone defects implanted with a high ratio of VEGF-expressing cells, we observed a much greater abundance of endothelial cells (i.e., CD31-positive cells). Alternatively, excessive VEGF may increase recruitment of osteoclasts into the bone regeneration sites and lead to excessive bone resorption. However, our data do not support this possibility, since tartrate-resistant acid phosphatase activity (a marker for osteoclasts) was similar in both low- and high-VEGF groups.

In summary, our combination of loss-of-function and gain-of-function approaches reveals that VEGF plays multiple important roles in bone formation and bone regeneration mediated through the endochondral ossification pathway. We suspect that the synergistic effect between angiogenic factor VEGF and osteogenic factor BMP4 should be more prominent in individuals suffering from compromised circulation, such as patients with diabetes or atherosclerosis. Furthermore, our novel finding regarding the early effects of VEGF and BMP4 on bone healing may open new avenues to improve bone regeneration through enhancing stem cell recruitment and cell survival at the bone regeneration site. Finally, our unique observation that the ratio of VEGF to BMP4 should be carefully controlled to generate beneficial effects and prevent detrimental reactions also has important implications for designing new tissue regeneration strategies that involve the use of VEGF and other growth factors.

\section{Acknowledgments}

We wish to thank Ryan Pruchnic for excellent technical assistance in imaging, David Stenger and Ryan Sauder for editorial assistance, and Dave Hannallah and Brett Young for helpful discussion. This work was supported in part by an NIH grant (1 R01 DE13420$01)$ to J. Huard, and by grants to H. Peng and J. Huard from the Pittsburgh Tissue Engineering Initiative, the Albert B. Ferguson, Jr., Orthopaedic Fund of The Pittsburgh Foundation. This work was also supported by the William F. and Jean W. Donaldson Chair at the Children's Hospital of Pittsburgh. 
1. Praemer, A., Furner, S., and Rice, D.P. 1992. Musculoskeletal conditions in the United States. Proceedings of the American Academy of Orthopaedic Surgeons. Park Ridge, Illinois, USA. 83-124.

2. Urist, M.R. 1965. Bone formation by autoinduction. Science 150:893-899.

3. Wozney, J.M., et al. 1988. Novel regulators of bone formation: molecular clones and activities. Science. 242:1528-1534.

4. Wang, E.A., et al. 1988. Purification and characterization of other distinct bone-inducing factors. Proc. Natl. Acad. Sci. USA. 85:9484-9488.

5. Harland, R.M. 1994. The transforming growth factor beta family and induction of the vertebrate mesoderm: bone morphogenetic proteins are ventral inducers. Proc. Natl. Acad. Sci. USA. 91:10243-10246.

6. Fang, J., et al. 1996. Stimulation of new bone formation by direct transfer of osteogenic plasmid genes. Proc. Natl. Acad. Sci. USA 93:5753-5758

7. Lieberman, J.R., et al. 1999. The effect of regional gene therapy with bone morphogenetic protein-2-producing bone-marrow cells on the repair of segmental femoral defects in rats. J. Bone Joint Surg. Am. 81:905-917.

8. Lou, J., Xu, F., Merkel, K., and Manske, P. 1999. Gene therapy: adenovirus-mediated human bone morphogenetic protein-2 gene transfer induces mesenchymal progenitor cell proliferation and differentiation in vitro and bone formation in vivo. J. Orthop. Res. 17:43-50.

9. Alden, T.D., et al. 1999. In vivo endochondral bone formation using a bone morphogenetic protein 2 adenoviral vector. Hum. Gene Ther. 10:2245-2253.

10. Lee, J.Y., et al. 2000. Clonal isolation of muscle-derived cells capable of enhancing muscle regeneration and bone healing. J. Cell Biol. 150:1085-1100.

11. Peng, H., et al. 2001. Development of an MFG-based retroviral vector system for secretion of high levels of functionally active human BMP4. Mol. Ther. 4:95-104.

12. Gazit, D., et al. 1999. Engineered pluripotent mesenchymal cells integrate and differentiate in regenerating bone: a novel cell-mediated gene therapy. J. Gene Med. 1:121-133.

13. Turgeman, G., et al. 2001. Engineered human mesenchymal stem cells: a novel platform for skeletal cell mediated gene therapy. J. Gene Med. 3:240-251.

14. Moutsatsos, I.K., et al. 2001. Exogenously regulated stem cell-mediated gene therapy for bone regeneration. Mol. Ther. 3:449-461.

15. Mori, S., et al. 1998. Antiangiogenic agent (TNP-470) inhibition of ectopic bone formation induced by bone morphogenetic protein-2. Bone. 22:99-105.
16. Gerber, H.P., et al. 1999. VEGF couples hypertrophic cartilage remodeling, ossification and angiogenesis during endochondral bone formation. Nat. Med. 5:623-628.

17. Wang, D.S., et al. 1996. Increase of vascular endothelial growth factor mRNA expression by 1,25-dihydroxyvitamin D3 in human osteoblastlike cells. J. Bone Miner. Res. 11:472-479.

18. Springer, M.L., Chen, A.S., Kraft, P.E., Bednarski, M., and Blau, H.M. 1998. VEGF gene delivery to muscle: potential role for vasculogenesis in adults. Mol. Cell. 2:549-558.

19. Lee, R.J., et al. 2000. VEGF gene delivery to myocardium: deleterious effects of unregulated expression. Circulation. 102:898-901.

20. Miquerol, L., Langille, B.L., and Nagy, A. 2000. Embryonic development is disrupted by modest increases in vascular endothelial growth factor gene expression. Development. 127:3941-3946.

21. Muller, W.A., Ratti, C.M., McDonnell, S.L., and Cohn, Z.A. 1989. A human endothelial cell-restricted, externally disposed plasmalemmal protein enriched in intercellular junctions. J. Exp. Med. 170:399-414.

22. Leung, D.W., Cachianes, G., Kuang, W.J., Goeddel, D.V., and Ferrara, N. 1989. Vascular endothelial growth factor is a secreted angiogenic mitogen. Science. 246:1306-1309.

23. Keck, P.J., et al. 1989. Vascular permeability factor, an endothelial cell mitogen related to PDGF. Science. 246:1309-1312.

24. Cho, N.K., et al. 2002. Developmental control of blood cell migration by the Drosophila VEGF pathway. Cell. 108:865-876.

25. Dias, S., Shmelkov, S.V., Lam, G., and Rafii, S. 2002. VEGF(165) promotes survival of leukemic cells by $\mathrm{Hsp} 90$-mediated induction of Bcl-2 expression and apoptosis inhibition. Blood. 99:2532-2540.

26. Hay, E., Lemonnier, J., Fromigue, O., and Marie, P.J. 2001. Bone morphogenetic protein-2 promotes osteoblast apoptosis through a Smadindependent, protein kinase C-dependent signaling pathway. J. Biol. Chem. 276:29028-29036.

27. Li, G., White, G., Connolly, C., and Marsh, D. 2002. Cell proliferation and apoptosis during fracture healing. J. Bone Miner. Res. 17:791-799.

28. Vu, T.H., et al. 2000. MMP-9/gelatinase B is a key regulator of growth plate angiogenesis and apoptosis of hypertrophic chondrocytes. Cell. 93:411-422.

29. Harada, S., et al. 1994. Induction of vascular endothelial growth factor expression by prostaglandin E2 and E1 in osteoblasts. J. Clin. Invest. 93:2490-2496.

30. Deckers, M.M., et al. 2000. Expression of vascular endothelial growth factors and their receptors during osteoblast differentiation. Endocrinology. 141:1667-1674. 